\title{
Modulated fiber ring laser and its application in high-sensitivity temperature sensors
}

\author{
R. A. Perez-Herrera*ab, S. Tainta ${ }^{\text {ab }}$, M. J. Erro and M. Lopez-Amo ab \\ apublic University of Navarra, Dept. of Electric and Electronic Engineering. Pamplona, Spain \\ ${ }^{\mathrm{b}}$ ISC - Institute of Smart Cities, Public University of Navarra, 31006, Pamplona, Spain
}

\begin{abstract}
This work presents an experimental demonstration of a scheme based on an internally modulated fiber ring laser for highsensitivity temperature sensing. The attained temperature resolution has been as low as $\pm 2 \mathrm{pm}$ even when a commercial FBG with a sensitivity of $10 \mathrm{pm} /{ }^{\circ} \mathrm{C}$ was used. Thus, a fivefold improvement in the temperature sensor resolution can be achieved when compared to a simple FBG interrogation scheme. In addition to this, the measuring range could be selected only by changing the frequency modulation of the fiber ring laser. This technology also allows to triple the photodiode bandwidth unambiguously when temperature or strain measurements are carried out, which is a remarkable achievement in term of cost reduction.
\end{abstract}

Keywords: fiber ring laser, temperature sensor, amplitude modulation, single-sideband modulation.

\section{INTRODUCTION}

Fiber optic temperature sensors have been deeply studied due to the fact that they offer many advantages such as durability in harsh environment, small size and weight and immunity to electromagnetic interference [1]. In addition to this, these sensors can be employed in very different applications such as aeronautics, health monitoring [2], or heat treatments for foods [3]. Nevertheless some applications require lower sensitivities that those obtained by using for example, fiber Bragg gratings (FBG). The Bragg wavelength shift is about $0.01 \mathrm{~nm} /{ }^{\circ} \mathrm{C}$ in silica, and a wavelength resolution of $10 \sim 11 \mathrm{pm}$ (at $1.5 \mu \mathrm{m})$ is required to resolve a temperature change of $1{ }^{\circ} \mathrm{C}$ due to the small thermal expansion coefficient and its small thermo-optic coefficient[4] . Because this thermal sensitivity is too small to discern a temperature change of $1^{\circ} \mathrm{C}$, a number of techniques have been considered for improving the temperature sensitivity of the FBGs: coating the cladding of the FBG with temperature-sensitive materials, cladding-etching or polymer packaging [5], [6].

In this work the initial results of a technique based on an internally modulated fiber ring lasers for high-sensitivity temperature measurement are presented. The proposed setup attains a temperature measurement resolution as low as $\pm 2 \mathrm{pm}$ when a commercial FBG with a sensitivity of $10 \mathrm{pm} /{ }^{\circ} \mathrm{C}$ is used as sensor, ensuing a resolution improvement of five times when compared to a simple interrogation scheme. The measurement range of the system is defined as three times the modulating frequency of the fiber ring laser, being possible to tune it just by modifying this parameter. Also, the maximum required bandwidth of the photodetector used is determined by the modulating frequency. Thus, this scheme enables the unambiguous measurement of temperature over a bandwidth which triples the available bandwidth of the photodiode employed, which is a remarkable achievement in terms of reducing costs.

\section{EXPERIMENTAL SETUP}

The schematic setup of the erbium doped fiber ring laser (EDFRL) used to carry out this experiment is shown in Fig. 1. This EDFRL comprises a standard single-mode fiber (SMF) ring within which a highly doped Er-fibre (M-12, with absorption of $16-20 \mathrm{~dB} / \mathrm{m}$ at $1531 \mathrm{~nm}$, by Fibercore) has been incorporated, acting as the active medium. The amplification was obtained by using 4 meters of this highly doped erbium fiber. The fiber length needed for the cavity was shorter than in other cases [7]. Additionally, a 980-nm pump source and a wavelength division multiplexer (WDM) were used to form the ring resonator.

The wavelength selection was carried out by means of a FBG with a Bragg wavelength of $1552.3 \mathrm{~nm}$ and the optical isolator was included to ensure unidirectional oscillation in the ring cavity avoiding the hole burning effect. A $3 \mathrm{~dB}$ coupler was

Sixth European Workshop on Optical Fibre Sensors, edited by Elfed Lewis, Proc. of SPIE Vol. 9916, 99160B · ( ) 2016 SPIE · CCC code: 0277-786X/16/\$18 · doi: 10.1117/12.2236847 
used to incorporate the FBG into the laser cavity. Between the coupler and the FBG, a four port optical circulator was placed in order to insert an amplitude modulator and a polarization controller (PC). In this experimental demonstration, the attained reflected signal from the FBG was modulated at $10 \mathrm{GHz}$. Finally, a $3 \mathrm{~dB}$ coupler was employed to extract part of the erbium doped fiber laser optical power from the ring to the output port. This laser line was combined with a reference obtained from the output of a commercial tunable laser source (TLS) with a linewidth of $100 \mathrm{KHz}$. The output signal was simultaneously monitored by an optical spectrum analyzer (OSA) with a highest spectral resolution of $0.01 \mathrm{~nm}$ and an electrical spectrum analyzer (ESA) whose resolution bandwidth can be as good as $1 \mathrm{~Hz}$. The photodetector used had a bandwidth of $38 \mathrm{GHz}$, although a bandwidth similar to the modulating frequency, that is, only $10 \mathrm{GHz}$ is required for the proposed scheme.

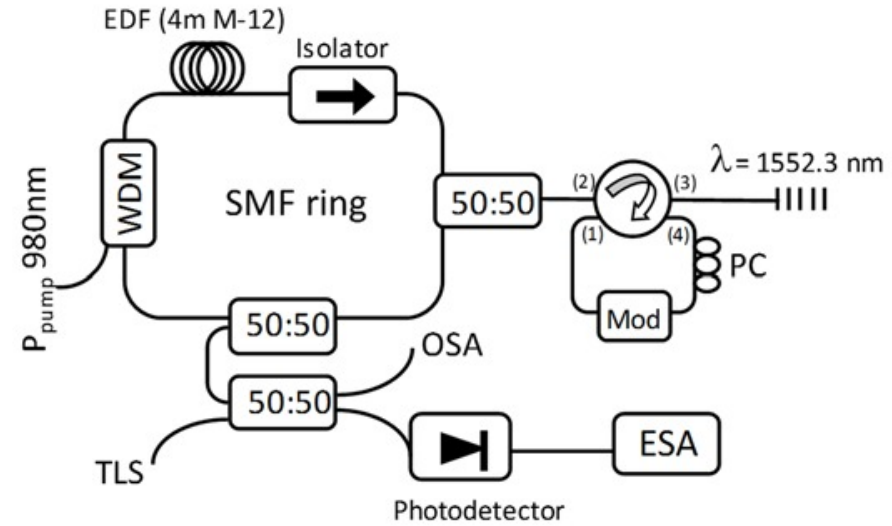

Figure 1. Proposed erbium doped fiber ring laser for high-sensitivity temperature sensor applications.

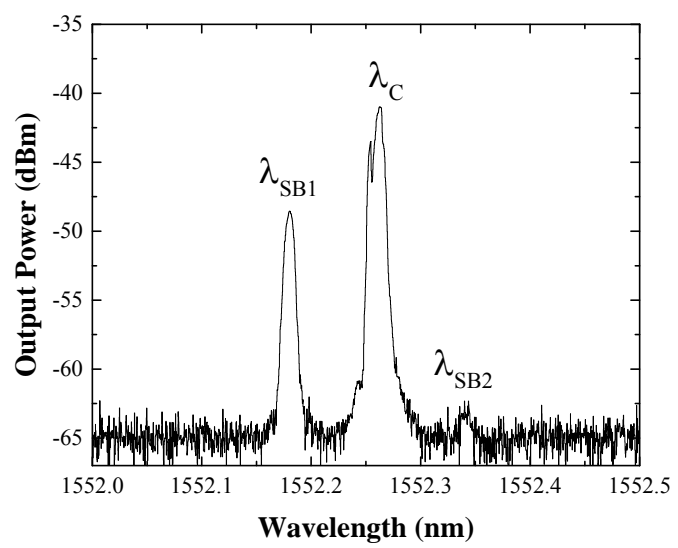

Figure 2._Output optical spectrum for the EDFRL modulated at $10 \mathrm{GHz}$ and where SSB was applied.

\section{RESULTS}

Figure 2 shows the output optical spectrum for this EDFRL for a 500mA pump power. In this case a single-sideband modulation (SSB) has been selected with a modulating frequency of $10 \mathrm{GHz}$. As can be seen in this figure, the SSB $\left(\lambda_{\mathrm{SB} 1}\right)$ and carrier $\left(\lambda_{C}\right)$ are centered at $1552.175 \mathrm{~nm}$ and $1552.256 \mathrm{~nm}$ respectively and the output power level are about $-47 \mathrm{dBm}$ and $-40 \mathrm{dBm}$ in that order. Likewise, second side band $\left(\lambda_{\mathrm{SB} 2}\right)$ does not appear in this optical spectrum. The characterization of this new laser for sensor application was carried out by changing the central wavelength of the TLS. By doing so, instead of testing the sensor by heating the FBG on a climatic chamber in a specific range, the TLS was tuned from $1552.175 \mathrm{~nm}$ (over $\lambda_{\mathrm{SB} 1}$ ) to $1552.287 \mathrm{~nm}$ in order to evaluate the span under test.

In this case, as the modulating frequency applied to the fiber ring laser was $10 \mathrm{GHz}$, the separation between these two peaks is about $0.08 \mathrm{~nm}$. Taking into consideration the fact that the typical temperature sensitivity of a FBG is about 10 $\mathrm{pm} /{ }^{\circ} \mathrm{C}$, the temperature range to be measured within this spectral range would be about $8^{\circ} \mathrm{C}$. This modulation frequency was selected to be $10 \mathrm{GHz}$ in order to be easily distinguished by the OSA resolution but the temperature range than can be evaluated could be decreased or increased only by changing this parameter according to our needs.

Figures 3 and 4 illustrate the optical and electrical results when the TLS was tuned to $1552.2 \mathrm{~nm}$. As figure 3 shows, the TLS $\left(\lambda_{\mathrm{TLS}}\right)$ was tuned closer to the SSB $\left(\lambda_{\mathrm{SB} 1}\right)$ than the carrier $\left(\lambda_{\mathrm{C}}\right)$. That fact can be also observed in the electrical domain (see Figure 4). The first observed beat signal, centered at around $3 \mathrm{GHz}$, corresponds to the beat between the TLS and the $\lambda_{\mathrm{SB} 1}$ because its output power lever is lower than the second one. This second beat signal, centered at about $7 \mathrm{GHz}$, was due to the beat between the TLS and the carrier $\left(\lambda_{C}\right)$. Finally, the third peak centered at $10 \mathrm{GHz}$ corresponds to the modulation introduced in our fiber ring laser. 


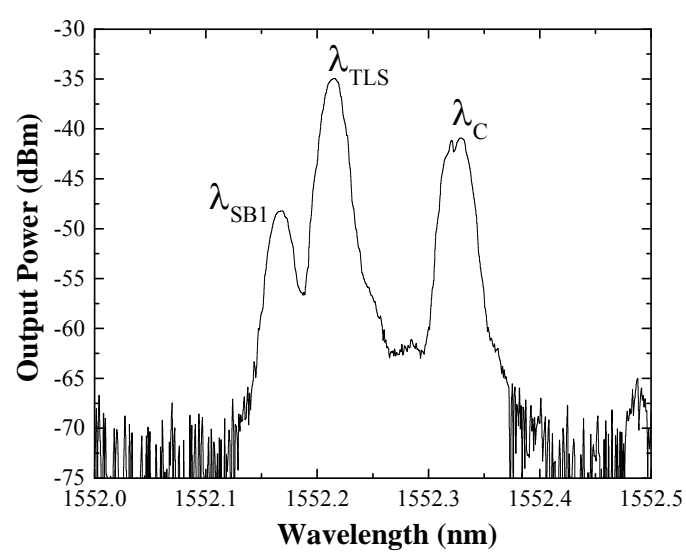

Figure 3. Output optical spectrum for the EDFRL modulated at $10 \mathrm{GHz}$ and the TLS tuned to be located at $1552.2 \mathrm{~nm}$.

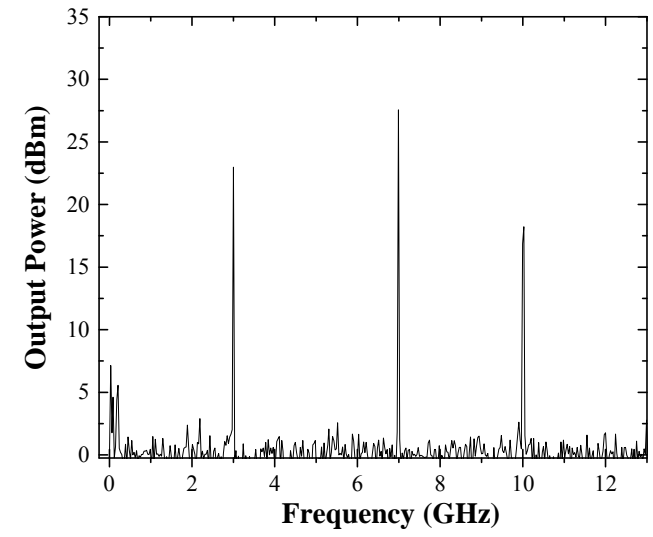

Figure 4. Output optical spectrum measured by the ESA for the EDFRL when the tunable laser was tuned to $1552.2 \mathrm{~nm}$.

Furthermore, if the TLS is tuned to be closer to $\lambda_{c}$, the output optical spectrum measured by the ESA shows opposite values of output power levels of the beating peaks as Figure 5 illustrates. Also to determine the temperature resolution, a measurement of one hour for each analyzed case using the max-hold mode at ESA, was carried out. The measure resolution corresponds to the difference between the higher and the lowest frequency. In order to evaluate the worst scenario, the TLS was tuned at exactly the same wavelength as one of the peaks (at $\lambda_{\mathrm{SB} 1}=1552.175 \mathrm{~nm}$ ) and a confidence level (CL) of $100 \%$ was consider. As can be shown at Fig. 6 , a minimum resolution of around $500 \mathrm{MHz}( \pm 2 \mathrm{pm})$ was retrieved, being these results better than the $15 \mathrm{pm}$ and $10 \mathrm{pm}$ obtained in [8] and [9], respectively. This resolution could be further enhanced by reducing the mode hopping inside the laser ring cavity.

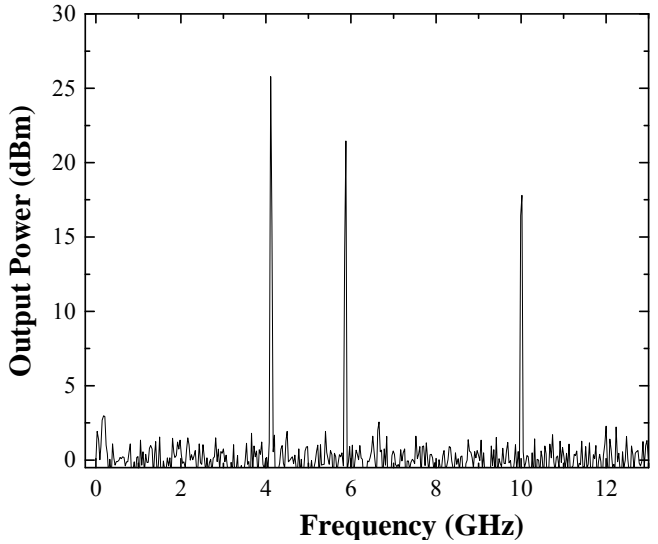

Figure 5. Output optical spectrum measured by the ESA for the EDFRL when the tunable laser was tuned to $1552.223 \mathrm{~nm}$.

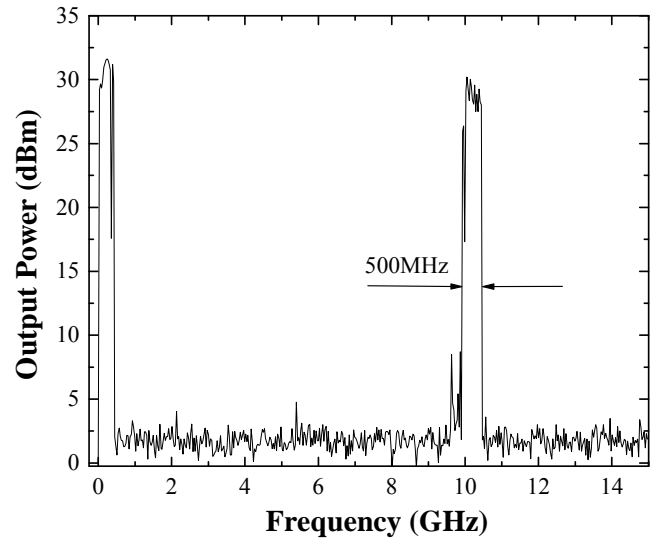

Figure 6. Frequency instability when the tunable laser was tuned to $1552.2 \mathrm{~nm}$ measured with Max-Hold mode in ESA

Additionally, in the case of the central wavelength of the TLS exceeding the carrier's wavelength (see Figure 7), the output optical spectrum measured by the OSA shows that this wavelength is out of the temperature measurement range. However, in this case there will still be a beating peak within the measurement bandwidth of the ESA, corresponding to the product of the carrier and the reference laser. For example, as it can be seen in figure 8, one beating peak still exists at around 4 $\mathrm{GHz}$. As a result of this, it will be possible to extend the measurement range $10 \mathrm{GHz}$ above the reference wavelength. Conversely, when the central wavelength of the TLS falls below the modulation sideband, the beating peak of the sideband and the TLS laser will fall within the measurement bandwidth, being possible to distinguish both cases due to the different power level of the beating signals. Thus, it is possible to extend the measurement range of the system unambiguously, allowing to triple the effective bandwidth of the photodiode. Using this technique, the temperature range to be measured with this technique is not about $8^{\circ} \mathrm{C}$, but $24^{\circ} \mathrm{C}$. 


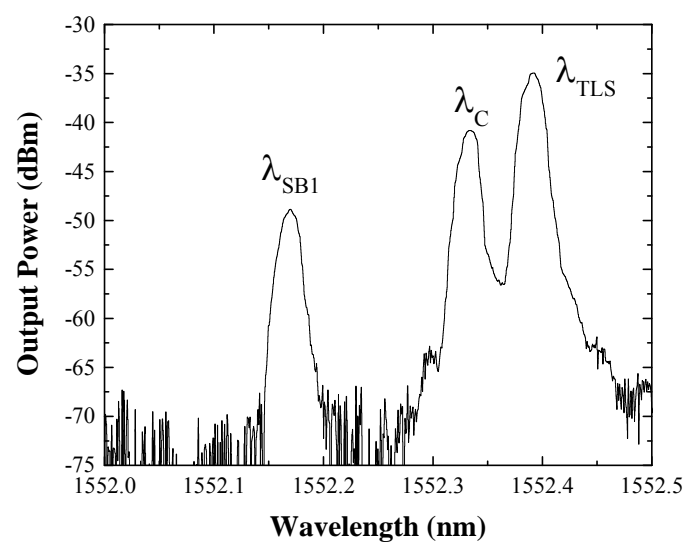

Figure 7. Output optical spectrum measured by the ESA when the TLS is tuned to be outside of the temperature measure range.

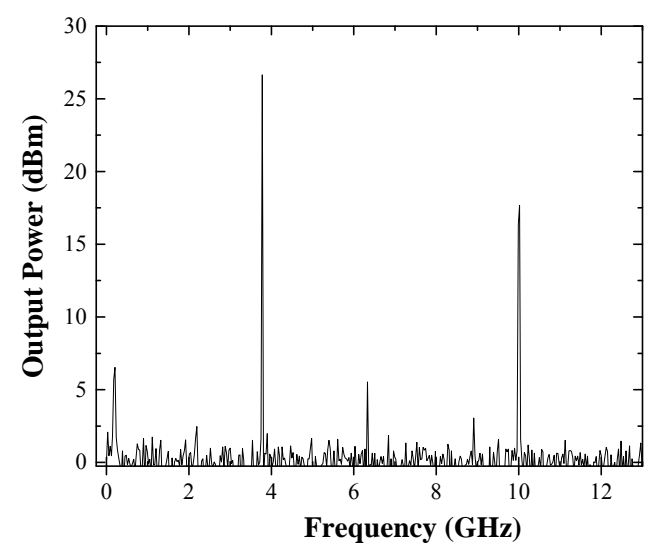

Figure 8. Output optical spectrum measured by the OSA when the TLS is tuned to be outside of the temperature measure range.

\section{CONCLUSIONS}

In this work it has been experimentally demonstrated a scheme based on a modulated fiber ring laser and its application in high-sensitivity temperature sensors. The attained resolution was as low as $\pm 2 \mathrm{pm}$ even when a commercial FBG with a sensitivity of $10 \mathrm{pm} /{ }^{\circ} \mathrm{C}$ is used as sensor. The measurement range of the system is defined as three times the modulating frequency of the fiber ring laser, being possible to tune it just by modifying this parameter. Also, the maximum required bandwidth of the photodetector used is determined by the modulating frequency. Thus, this scheme enables the unambiguous measurement of temperature over a bandwidth which triples the available bandwidth of the photodiode employed, which is a remarkable achievement in terms of reducing costs.

\section{ACKNOWLEDGMENT}

This work was supported by the Spanish Government projects TEC2013-47264-C2-2-R and TEC2014-58048-C2-1-P.

\section{REFERENCES}

1. Marlen A. Gonzalez-Reyna, Edgar Alvarado-Mendez, et. Al, "Laser Temperature Sensor Based on a Fiber Bragg Grating," IEEE Photonics Technology Letters 27(11), 1141-1144 (2015)

2. Yu, Q., Zhang, Y., Dong, Y., Li, Y. P., Wang, C. and Chen, H., "Study on optical fiber Bragg grating temperature sensors for human body temperature monitoring," Proc. IEEE Symp. Photonics and Optoelectronics (SOPO), 1-4 (2012)

3. Sosa-Morales, M. E., Rojas-Laguna, R. and López-Malo, A., "Optical fiber temperature sensors: Applications in heat treatments for foods," Proc. SPIE. 7839, 78391L, (2010)

4. Cherl-Hee Lee, Jonghun Lee, Min-Kuk Kim and Kwang Taek Kim "Characteristics of a Fiber Bragg Grating Temperature Sensor Using the Thermal Strain of an External Tube," J. Korean Phys. Soc. 59(5), 3188-3191 (2011)

5. Paul, J., Ngoi, B.K.A., Zhao, L.P. and Fang, Z.P., "Enhanced Lateral Pressure Tuning of Fiber Bragg Grating by polymer packaging," Optics Communications 242(4-6), 425-430 (2004)

6. Chen, X., Zhou, K., Zhang L. and Bennion, I. "Simultaneous measurement of temperature and external refractive index by use of a hybrid grating in D fiber with enhanced sensitivity by HF etching," Appl. Opt. 44, 178 (2005).

7. Talaverano, L., Abad, S., Jarabo, S. and López-Amo, M. "Multiwavelength fiber laser sources with Bragg-grating sensor multiplexing capability," J. Lightw. Technol. 19(4), 553-558 (2001)

8. Perez-Herrera, R. A. Quintela, M. A., Fernandez-Vallejo, M., Quintela, A., Lopez-Amo, M. et. al "Stability comparison of two ring resonator structures for multiwavelength fiber lasers using highly doped er-fibers," J. Lightw. Technol. 27(14), 2563-2569 (2009)

9. Zhang K. and Kang J. U., "C-band wavelength-swept single-longitudinal mode erbium-doped fiber ring laser," Opt. Exp. 16(18), 14173-14179 (2008). 